Research Paper

\title{
Free Edge Effects in Sandwich Laminates Under Tension, Bending and Twisting Loads
}

\author{
$\mathrm{N}$ DHANESH $^{1}$ and SANTOSH KAPURIA ${ }^{1,2 *}$ \\ ${ }^{1}$ Department of Applied Mechanics, Indian Institute of Technology Delhi, New Delhi 110 016, India \\ ${ }^{2}$ CSIR-Structural Engineering Research Centre, Taramani, Chennai 600113 India
}

(Received on 20 April 2016; Accepted on 28 April 2016)

\begin{abstract}
An accurate analytical solution for predicting the free edge effects in sandwich laminates under tension, bending and twisting loading is presented. The recently developed mixed-field multiterm extended Kantorovich method (MMEKM) has been used to obtain the solution of the governing equations, which are developed using the Reissner-type variational principle. The present mixed-field approach enables the exact and point-wise satisfaction of traction-free edge and interlaminar continuity conditions for displacements and stresses. The numerical results presented for different loadings and lay-up show rapid convergence of the iterative series solution. The comparison of the present results with the detailed FE solution shows good agreement. The present solution captures the singularity of stresses in the free edge region by showing the rise in its peak magnitude with the number of terms in the solution. The presented accurate 3D elasticity based solution can act as a useful benchmark for assessing the accuracy of solutions obtained from other approximate methods.
\end{abstract}

Keywords: Free Edge Effects; Sandwich Laminates; Extended Kantorovich Method; 3D Elasticity; Mixed Formulation

\section{Introduction}

With the wide use of laminated composite and sandwich structures in many advanced applications (aerospace, automobile, naval, civil etc.), the vulnerability of such structures to delamination damage initiating from the edge region has been a serious concern among the designers. The occurrence of localized interlaminar stresses near the free edge/ boundary region is known to be the main reason for initiation of such damage, and is caused by the material and geometric discontinuities that exist at the interlayer regions at the free edge boundaries. The development of such three dimensional (3D) stresses in the vicinity of free edges under various loading conditions are commonly known as the free edge effect, and has been a topic of intense research since the work of Hayashi (1967). In this work, an accurate analytical 3D elasticity based solution for the free edge stress field in sandwich laminates under axial extension, bending and twisting loadings is presented.
Comprehensive reviews of various methodologies used by researchers for studying the free edge effects have been reported by Mittelstedt and Becker $(2004,2007)$ and Kant and Swaminathan (2000). Subsequent to the initial work of Hayashi (1967) and Puppo and Evensen (1970), where they presented approximate solutions for transverse interlaminar shear stresses by neglecting the transverse normal stress, Pipes and Pagano (1970) presented a finite difference (FD) solution of the complete system of 3D elasticity equations for the free edge problem. Thereafter, there has been a continuous effort to obtain accurate solutions for the free edge problem based on 3D elasticity, satisfying all boundary and interfacial conditions exactly at all points.

Various numerical methods such as the finite element (FE) method (Wang and Crossman, 1977; Raju and Crews, 1981; Lessard et al., 1996), the boundary element method (Davi and Milazzo, 1999) 
and the scaled boundary finite element method (Lindemann and Becker, 2000) have been employed for the free edge problems. The limitations of such numerical methods in accurately predicting the stress field in presence of sharp gradients and possible singularities are well known. Various approximate analytical/semi-analytical solutions have been presented, to overcome these issues. Cho and Yoon (1999) extended the Lekhnitskii stress function based solution of Flanagan (1994) for the free edge stresses in composite laminates under extension loading, employing the iterative extended Kantorovich method (EKM) (Kerr, 1968). This method has been further extended to obtain the free edge stress solution for symmetric laminates under bending, twisting and thermal loadings (Cho and Kim, 2000). In another development, Andakhshideh and Tahani (2013a,b) adopted a displacement based formulation in conjunction with the multiterm EKM for the free edge stress analysis of finite rectangular plates under extension, shear, bending, twisting and thermal loadings. The stress based formulations fail to satisfy pointwise interlaminar continuity conditions for displacements. On the other hand, in case of displacement based formulations, the interlaminar stress continuity and traction free edge conditions are not satisfied exactly at all points, but in an average sense. In both formulations, therefore the accuracy of predicted interlaminar stresses becomes questionable. Recently, the author group has presented a mixed-field multiterm EKM (MMEKM) solution for the free edge stress analysis of composite laminates under thermomechanical loadings (Dhanesh et al., 2016). The governing equations are developed using the Reissner-type mixed variational principle for composite laminates, considering both displacements and stresses as unknown variables. This approach allows exact satisfaction of the free edge traction free conditions as well as interlaminar continuity conditions of displacements and stresses in a point-wise sense. It also ensures the same degree of accuracy of the displacements and stresses.

All of the above mentioned studies on free edge stress analysis deal with composite laminates. Very few studies, however exist on the free edge effect in sandwich structures, which consist of relatively thin and stiff face sheets separated by a relatively soft thick and lightweight core. Such structures are preferred in applications where a higher bending stiffness is required, maintaining the light weight of the structure. Because of widely different material properties of the face sheet and core, the 3D elasticity solution may face numerical difficulties in solving for sandwich laminates. Lovinger and Frostig (2004) presented a hybrid approach for the study of free edge effects in soft core sandwich plates which is supported only at the lower face sheet, employing the classical laminate theory (CLT) approximations for the face sheets and 3D elasticity theory for the core. The analytical solution for bending, thermal, moisture loading conditions was obtained by using the EKM. Afshin et al. (2010) employed Reddy's layerwise theory (LWT) to study the free edge effects in cylindrical sandwich panel. Recently, a closed-form solution for the free edge stress field in sandwich structure subjected to differential temperature and mechanical loading has been presented by Wong $(2015,2016)$, following the strength of material approach. In this work, the face sheets are modelled as beam elements and the soft core as an elastic medium. The approach leads to a discontinuous peeling stress at the interface between core and face sheet layers. Such simplified 2D theory based solutions generally lead to inaccurate prediction of the 3D free edge stress field. In the present work, an accurate solution for the free edge stress field in sandwich laminates under tension, bending and twisting loadings is presented using the recently developed technique, MMEKM, of the author group (Dhanesh et al., 2016). The convergence of the iterative series solution and its comparison with the detailed FE analysis are presented. The results are obtained for sandwich laminates having both cross-ply and angle-ply lay-ups for the face sheets.

\section{Governing Equations}

\section{Reissner-type Mixed Variational Principle}

To study the free edge effect, an elastic sandwich panel having stiff unidirectional composite faces and a soft core is considered. The infinitely long ( $y$ direction) panel has a width $a$ in the $x$-direction and thickness $h$ in the $z$-direction. The panel has free edges at $x=0$ and $x=a$. It is subjected to a uniform axial strain $\left(\varepsilon_{0}\right)$, bending $\left(\chi_{0}\right)$, and twisting curvature $(\Theta)$ as shown in Fig. 1. The reference $x y$-plane of the $L$-layered panel is located at the mid-surface of the panel. The layers of the panel are numbered from 


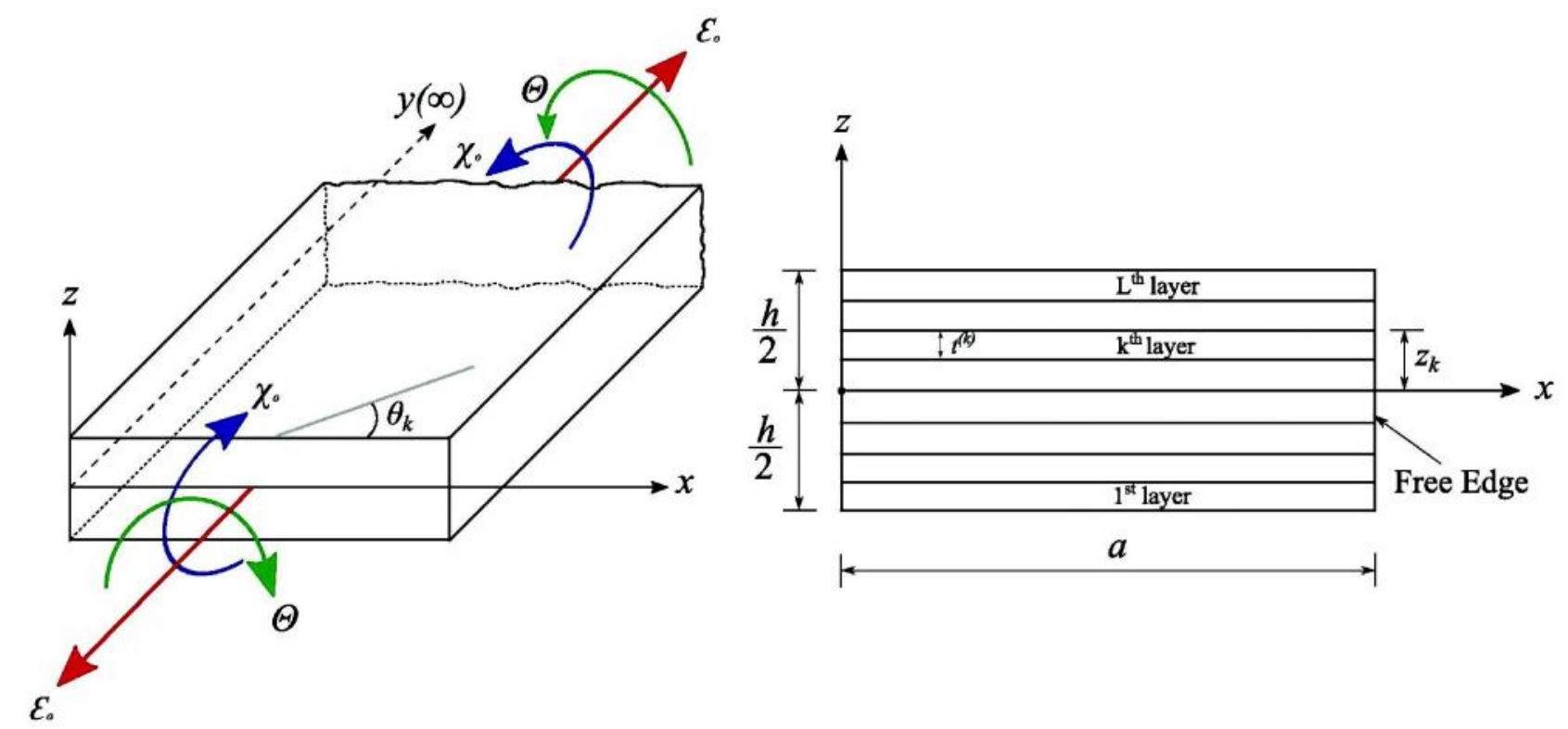

Fig. 1: Geometry of the sandwich laminated panel with free edges subjected to extension, bending and twisting loading

bottom to top, and the $z$-coordinate of the upper surface of the $k$ th layer with respect to the $x y$-plane is denoted as $z_{k}$. The thickness of each layer can be different, and for $k$ th layer it is denoted as $t^{(k)}$. The fibres of the unidirectional composite laminas are oriented at an angle $\theta$ with the $x$-axis. The principal material axis $x_{3}$ of all the layers is oriented along the $z$-direction.

Since the problem falls under the class of generalized plane deformation as described by Lekhnitskii (1963), the displacement field $u_{i}(x, y, z)$ in the laminate under extension, bending and twisting loading can be written as

$$
\begin{aligned}
& u_{1}=u(x, z)-\Theta y z+\omega_{2} z-\omega_{3} y+u_{0} \\
& u_{2}=v(x, z)-\left(\varepsilon_{0}-\chi_{0} z\right) y+\omega_{3} x-\omega_{1} z+v_{0} \\
& u_{3}=w(x, z)+\frac{\chi_{0} y^{2}}{2}+\Theta x y+\omega_{1} y-\omega_{2} x+w_{0}
\end{aligned}
$$

where $u_{i}(i=1,2,3)$ are the displacement components in $x, y$ and $z$ direction, respectively. $u, v$ and $w$ are the unknown displacements, which are functions of $x$ and $z$ coordinates. The constants $u_{0}, v_{0}, w_{0}$ and $\omega_{1}, \omega_{2}, \omega_{3}$ characterize the rigid body translations and rotations of the panel, respectively.
Using the displacement field given in Eq. (1), the normal and shear strains, $\varepsilon_{i}$ and $\gamma_{i j}$ can be obtained as

$$
\begin{aligned}
& \varepsilon_{x}=u_{, x}, \varepsilon_{y}=\varepsilon_{0}-\chi_{0} z, \varepsilon_{z}=w_{, z}, \\
& \gamma_{y z}=v_{, z}+\Theta x, \gamma_{z x}=w_{, x}+u_{, z}, \gamma_{x y}=v_{, x}-\Theta z
\end{aligned}
$$

where a subscript comma followed by $x$, for example, denotes partial differentiation with respect to $x$.

For the $k$ th layer, the constitutive relationship between the strain $\left(\varepsilon_{i j}\right)$ and stress $\left(\sigma_{i j}\right)$ components in the plate coordinate system $(x, y, z)$ can be expressed as

$$
\left[\begin{array}{l}
\varepsilon_{x} \\
\varepsilon_{y} \\
\varepsilon_{z} \\
\gamma_{y z} \\
\gamma_{z x} \\
\gamma_{x y}
\end{array}\right]=\left[\begin{array}{llllll}
\bar{s}_{11} & \bar{s}_{12} & \bar{s}_{13} & 0 & 0 & \bar{s}_{16} \\
\bar{s}_{12} & \bar{s}_{22} & \bar{s}_{23} & 0 & 0 & \bar{s}_{26} \\
\bar{s}_{13} & \bar{s}_{23} & \bar{s}_{33} & 0 & 0 & \bar{s}_{36} \\
0 & 0 & 0 & \bar{s}_{44} & \bar{s}_{45} & 0 \\
0 & 0 & 0 & \bar{s}_{45} & \bar{s}_{55} & 0 \\
\bar{s}_{16} & \bar{s}_{26} & \bar{s}_{36} & 0 & 0 & \bar{s}_{66}
\end{array}\right]\left[\begin{array}{l}
\sigma_{x} \\
\sigma_{y} \\
\sigma_{z} \\
\tau_{y z} \\
\tau_{z x} \\
\tau x y
\end{array}\right]
$$

where $\bar{s}_{i j}$ are the transformed elastic compliances, which can be expressed in terms of the engineering properties, namely, Young's moduli $Y_{i}$, shear moduli $G_{i j}$ and major Poisson's ratios $\nu_{i j}$ (Jones, 1999). Upon 
substitution of $\varepsilon_{y}$ from Eq. (2) into the corresponding constitutive relation in Eq. (3), $\sigma_{y}$ is obtained as

$$
\begin{aligned}
\sigma_{y}= & \left(\varepsilon_{0}-\chi_{0} z\right) / \bar{s}_{22}-\left(\bar{s}_{12} / \bar{s}_{22}\right) \sigma_{x} \\
& -\left(\bar{s}_{23} / \bar{s}_{22}\right) \sigma_{z}-\left(\bar{s}_{26} / \bar{s}_{22}\right) \tau_{x y}
\end{aligned}
$$

Substituting the above expression, $\sigma_{y}$ can be eliminated from the other constitutive relations in Eq. (3) as

$$
\begin{aligned}
& \varepsilon_{x}=p_{11} \sigma_{x}+p_{13} \sigma_{z}+p_{16} \tau_{x y}+\tilde{s}_{21}\left(\varepsilon_{0}-\chi_{0} z\right), \\
& \varepsilon_{z}=p_{31} \sigma_{x}+p_{33} \sigma_{z}+p_{36} \tau_{x y}+\tilde{s}_{23}\left(\varepsilon_{0}-\chi_{0} z\right) \\
& \gamma_{x y}=p_{61} \sigma_{x}+p_{63} \sigma_{z}+p_{66} \tau_{x y}+\tilde{s}_{26}\left(\varepsilon_{0}-\chi_{0} z\right)
\end{aligned}
$$

where $p_{i j}=\bar{s}_{i j}-\bar{s}_{2 i} \bar{s}_{2 j} / \bar{s}_{22}, \tilde{s}_{2 j}=\bar{s}_{2 j} / \bar{s}_{22}$, for $i, j=$ $1,3,6$. The governing equations for the free edge problem are developed using the Reissner-type variational principle (Shames and Dym, 1985) for linear elastic medium, which can be written as

$$
\begin{aligned}
& \quad \int_{v}\left[\delta u\left(\tau_{z x, z}+\sigma_{x, x}\right)\right. \\
& +\delta v\left(\tau_{y z, z}+\tau_{x y, x}\right)+\delta w\left(\sigma_{z, z}+\tau_{z x, x}\right) \\
& +\delta \sigma_{x}\left(\varepsilon_{x}-u_{, x}\right)+\delta \sigma_{z}\left(\varepsilon_{z}-w_{, z}\right) \\
& \quad+\delta \tau_{y z}\left(\gamma_{y z}-v_{, z}\right) \\
& \quad+\delta \tau_{z x}\left(\gamma_{z x}-u_{, z}-w_{, x}\right) \\
& \left.\quad+\delta \tau_{x y}\left(\gamma_{x y}-v_{, x}\right)\right] d V \\
& -\int_{A_{T}}\left(T_{i}^{n}-\bar{T}_{i}^{n}\right) \delta u_{i} d A-\int_{A_{u}} T_{i}^{n} \delta u_{i} d A=0, \forall \delta u_{i}, \delta \sigma_{i}, \delta \tau_{i j}
\end{aligned}
$$

where $V$ denotes the volume of the panel per unit length. The summation convention for repeated indices holds for $i$ and $j . A_{T}$ and $A_{u}$ denote, respectively, the surface boundaries where tractions $\bar{T}_{i}^{n}$ and displacements $\bar{u}_{i}$ are prescribed. $T_{i}^{n}$ are the components of the traction $T_{i}$, given by $T_{i}^{n}=\sigma_{i j} n_{j}$, where $n_{j}$ denotes the direction cosines of the outward normal $\bar{n}$ to the surface. The area integral terms in Eq. (6) vanish, as all the surface boundary conditions are sought to be satisfied exactly. Upon substitution of the strain field obtained from strain-displacement relations in Eq. (1) and constitutive relations in Eqs. (3) and (5), the variational statement in Eq. (6) reads

$$
\int_{v}\left[\delta u\left(\tau_{z x, z}+\sigma_{x, x}\right)\right.
$$

$$
\begin{aligned}
& +\delta v\left(\tau_{y z, z}+\tau_{x y, x}\right)+\delta w\left(\sigma_{z, z}+\tau_{z x, x}\right) \\
& +\delta \sigma_{x}\left(p_{11} \sigma_{x}+p_{13} \sigma_{z}+p_{16} \tau_{x y}+\tilde{s}_{21}\left(\varepsilon_{0}-\chi_{0} z\right)-u_{, x}\right) \\
& +\delta \sigma_{z}\left(p_{31} \sigma_{x}+p_{33} \sigma_{z}+p_{36} \tau_{x y}+\tilde{s}_{23}\left(\varepsilon_{0}-\chi_{0} z\right)-w_{, z}\right) \\
& +\delta \tau_{y z}\left(\bar{s}_{44} \tau_{y z}+\bar{s}_{45} \tau_{z x}-v_{, z}-\Theta x\right) \\
& +\delta \tau_{z x}\left(\bar{s}_{45} \tau_{y z}+\bar{s}_{55} \tau_{z x}-u_{, z}-w_{, x}\right) \\
& +\delta \tau_{x y}\left(p_{61} \sigma_{x}+p_{63} \sigma_{z}+p_{66} \tau_{x y}\right. \\
& \left.\left.+\tilde{s}_{26}\left(\varepsilon_{0}-\chi_{0} z\right)-v_{, x}+\Theta z\right)\right] d V=0, \forall \delta u_{i}, \delta \sigma_{i}, \delta \tau_{i j}
\end{aligned}
$$

\section{Boundary and Interface Conditions}

The boundary conditions associated with the free edge problem considered in the present study are the traction free conditions at the bottom and top surfaces of the laminate and at the free edges, and the interlaminar continuity conditions assuming a perfect bonding between the layers at the interfaces. These conditions can be written as:

(i) traction free conditions at the bottom and top surfaces $(z=\mp h / 2)$ :

$$
\sigma_{z}=0, \tau_{y z}=0, \tau_{z x}=0
$$

(ii) continuity of displacements and stresses at the interface between the $k$ th and $(k+1)$ th layers

$$
\begin{aligned}
& \left.\left(u, v, w, \sigma_{z}, \tau_{y z}, \tau_{z x}\right)\right|_{z=z_{k}} ^{(k)} \\
& =\left.\left(u, v, w, \sigma_{z}, \tau_{y z}, \tau_{z x}\right)\right|_{z=z_{k}} ^{(k+1)}
\end{aligned}
$$

(iii) traction free conditions at the free edges at $x=$ $0, a$ :

$$
\sigma_{x}=0, \tau_{z x}=0, \tau_{x y}=0
$$

\section{MMEKM Solution of Governing Equations}

The MMEKM solution considers both displacements and stresses as primary variables. The field variable vector is defined as

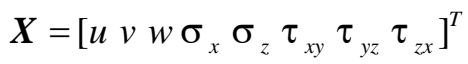


The following normalized coordinates $\xi$ and $\zeta^{(k)}$ are introduced for the in-plane and local thickness coordinate for the $k$ th layer such that they vary from 0 to 1 for $0 \leq x \leq a$ and $z_{k-1} \leq z \leq z_{k}$, respectively:

$$
\xi=x / a, \quad \zeta^{(k)}=\left(z-z_{k-1}\right) / t^{(k)}
$$

The solution of the field variables $X_{l}(\xi, \zeta)$ is expressed as an $n$-term series of the product of two independent functions $f_{l}^{i}(\xi)$ and $g_{l}^{i}(\zeta)$ in the inplane and thickness direction, respectively.

$$
X_{l}(\xi, \zeta)=\sum_{i=1}^{n} f_{l}^{i}(\xi) g_{l}^{i}(\zeta)
$$

Functions $g_{l}^{i}(\zeta)$ are defined separately for each layer, whereas functions $f_{l}^{i}(\xi)$ are the same for all layers. The analytical solutions for these functions are determined iteratively, satisfying all the boundary and interlaminar conditions specified in the previous section. Each iteration process involves two basic steps, which are described below.

\section{Step 1: Solving for Functions $g_{l}^{i}(\zeta)$}

In the first step of an iteration, functions $f_{l}^{i}(\xi)$ are treated as known from the previous iteration, and the functions $g_{l}^{i}(\zeta)$ for each layer are determined. In the first iteration, the following trigonometric functions are chosen as initial guess for $f_{l}^{i}(\xi)$ :

$$
\begin{aligned}
& f_{1}^{i}(\xi)=f_{2}^{i}(\xi)=f_{7}^{i}(\xi)=f_{8}^{i}(\xi)=\cos i \pi \xi \\
& f_{3}^{i}(\xi)=f_{4}^{i}(\xi)=f_{5}^{i}(\xi)=f_{6}^{i}(\xi)=\sin i \pi \xi
\end{aligned}
$$

Unlike other approximate methods of solving PDEs (e.g. Ritz and Galerkin), the EKM does not warrant the initial functions to satisfy the prescribed boundary conditions and the selection of initial functions does not have any adverse effect on the accuracy of the final solution. Since the first step considers $f_{l}^{i}(\xi)$ as known, the variation $\delta X_{l}$ obtained from Eq. (13) reads

$$
\delta X_{l}=\sum_{i=1}^{n} f_{l}^{i}(\xi) \delta g_{l}^{i}
$$

The unknown variables in the first step, $g_{l}^{i}(\zeta)$ for each layer are divided into two groups $\overline{\mathbf{G}}$ and $\hat{\mathbf{G}}$ as follows:

$$
\begin{aligned}
& \overline{\mathbf{G}}=\left[\begin{array}{llllllllllll}
g_{1}^{i} \ldots & g_{1}^{n} \ldots & g_{2}^{i} \ldots & g_{2}^{n} & g_{3 \ldots}^{i} & g_{3}^{n} & g_{5}^{i} \ldots & g_{5}^{n} g_{7}^{i} \ldots & g_{7}^{n} & g_{8}^{i} \ldots & g_{8}^{n}
\end{array}\right]^{\mathrm{T}} \\
& \hat{\mathbf{G}}=\left[\begin{array}{llll}
g_{4}^{i} \ldots & g_{4}^{n} & g_{6 \ldots}^{i} & g_{6}^{n}
\end{array}\right]^{T}
\end{aligned}
$$

where $\overline{\mathbf{G}}$ contains those displacements and stress components appearing in free edge boundary and interlaminar conditions [Eqs. (8) and (9)], and $\hat{\mathbf{G}}$ which contains the remaining two stress components, which are the dependent variables. Now, substitute $X_{l}$ and its variation $\delta X_{l}$ from (13) and (15) into the variational equation (7), and perform integration over the $\xi$-direction. Since the variations $\delta g_{l}^{i}$ are arbitrary, the coefficients of $\delta g_{l}^{i}$ in the integral must vanish individually, which results in the following first order differential-algebraic system of equations for each layer

$$
\begin{aligned}
& \mathbf{M} \overline{\mathbf{G}}_{, \zeta}=\overline{\mathbf{A}} \overline{\mathbf{G}}+\hat{\mathbf{A}} \hat{\mathbf{G}}+\overline{\mathbf{Q}} \\
& \mathbf{K} \hat{\mathbf{G}}=\tilde{\mathbf{A}} \overline{\mathbf{G}}+\tilde{\mathbf{Q}}
\end{aligned}
$$

where matrices $\mathbf{M}, \overline{\mathbf{A}}, \hat{\mathbf{A}}, \mathbf{K}$ and $\tilde{\mathbf{A}}$ are of size $6 n \mathbf{x}$ $6 n, 6 n \times 6 n, 6 n \times 2 n, 2 n \times 2 n$ and $2 n \times 6 n$, respectively. The nonzero elements of these matrices are identical to those presented in Dhanesh et al. (2016), and are omitted here for brevity. $\overline{\mathbf{Q}}, \tilde{\mathbf{Q}}$ are the load vectors of size $6 n$ and $2 n$, respectively. The nonzero elements of these load vectors are defined using the notation, $\langle\cdots\rangle_{a}=a \int_{0}^{1}(\cdots) d \xi$, which denotes the integration over the span length $a$ :

$$
\begin{aligned}
& \bar{Q}_{i 2}=-\Theta a t\left\langle\xi f_{7}^{i}\right\rangle_{a}, \bar{Q}_{i 3}=t \tilde{s}_{23}\left\langle f_{5}^{i}\right\rangle_{a}\left(\bar{\varepsilon}_{0}^{k}-\chi_{0} t \zeta\right), \\
& \tilde{Q}_{i 1}=-\tilde{s}_{21}\left\langle f_{4}^{i}\right\rangle_{a}\left(\bar{\varepsilon}_{0}^{k}-\chi_{0} t \zeta\right), \\
& \tilde{Q}_{i 2}=-\tilde{s}_{26}\left\langle f_{6}^{i}\right\rangle_{a}\left(\bar{\varepsilon}_{0}^{k}-\chi_{0} t \zeta\right)-\Theta\left\langle f_{6}^{i}\right\rangle_{a}\left(z_{k-1}+t \zeta\right)
\end{aligned}
$$


where $\bar{\varepsilon}_{0}^{k}=\varepsilon_{0}-\chi_{0} z_{k-1}$. All integrals appearing in the elements of the matrices in Eqs. (17)-(19) are evaluated exactly in closed form. The following $6 n$ first order ODEs in $\zeta$ are obtained after eliminating $\hat{\mathbf{G}}$ (obtained from Eq. (18)) from Eq. (17)

$$
\overline{\mathbf{G}}_{\zeta}=\mathbf{A} \overline{\mathbf{G}}+\mathbf{Q}
$$

where

$$
\mathbf{A}=\mathbf{M}^{-1}\left[\overline{\mathbf{A}}+\hat{\mathbf{A}} \mathbf{K}^{-1} \tilde{\mathbf{A}}\right] \text { and } \mathbf{Q}=\mathbf{M}^{-1}\left[\overline{\mathbf{Q}}+\hat{\mathbf{A}} \mathbf{K}^{-1} \tilde{\mathbf{Q}}\right]
$$

The solution of the above first order ODEs can be obtained analytically in closed form, and the general solution can be expressed in terms of $6 n$ real constants $C_{i}^{(k)}$ as

$$
\overline{\mathbf{G}}(\zeta)=\sum_{i=1}^{6 n} \mathbf{F}_{i}(\zeta) C_{i}^{(k)}+\mathbf{U}_{\mathbf{0}}+\zeta \mathbf{U}_{\mathbf{1}}
$$

where the elements of the column vector $F_{i}(\zeta)$ are expressed using the exponential and trigonometric functions of $\zeta$ in terms of the eigenvalues and eigenvectors of $A \cdot \mathbf{U}_{\mathbf{0}}$ and $\mathbf{U}_{\mathbf{1}}$ are the particular solution vectors corresponding to the constant and linear loading terms of $\mathbf{Q}$. The detailed solution procedure is omitted here for brevity and the same can be found in Kapuria and Kumari (2011). The $6 n$ x $L$ unknown constants $C_{i}^{(k)}$ 's for $L$ layers are determined from the following $6 n$ surface boundary and $6 n \times(L-1)$ interface conditions, obtained from Eqs. (8) and (9):

$$
\begin{aligned}
& \text { for } k=1 \text { at } \zeta=0: g_{5}^{i}=0, g_{7}^{i}=0, g_{8}^{i}=0, \\
& \text { for } k=L \text {, at } \zeta=1: g_{5}^{i}=0, g_{7}^{i}=0, g_{8}^{i}=0 \\
& \left.\left(g_{1}^{i}, g_{2}^{i}, g_{3}^{i}, g_{5}^{i}, g_{7}^{i}, g_{8}^{i}\right)\right|_{\zeta=1} ^{(k)} \\
& \qquad=\left.\left(g_{1}^{i}, g_{2}^{i}, g_{3}^{i}, g_{5}^{i}, g_{7}^{i}, g_{8}^{i}\right)\right|_{\zeta=1} ^{(k+1)}
\end{aligned}
$$

for $k=1,2, \ldots(L-1)$ and $i=1,2, \ldots n$. The solution $\overline{\mathbf{G}}(\zeta)$ obtained after solving the system of ODEs is now substituted back into Eq. (18) to yield $\hat{\mathbf{G}}(\zeta)$. This completes the determination of functions $g_{l}^{i}(\zeta)$ for all the $L$ layers, and concludes the first step in an iteration process.

\section{Step 2: Solving for Functions $f_{l}^{i}(\xi)$}

In the second step of iteration, the solution for functions in the in-plane direction, $f_{l}^{i}(\xi)$ is obtained. Here, the solution for $g_{l}^{i}(\zeta)$ obtained in the previous step is considered as known, hence the variation $\delta X_{l}$ obtained from Eq. (13) reads

$$
\delta X_{l}=\sum_{i=1}^{n} g_{l}^{i}(\zeta) \delta f_{l}^{i}
$$

Similar to $g_{l}^{i}(\zeta)$, functions $f_{l}^{i}(\xi)$ also divided into two groups $\overline{\mathbf{F}}$ and $\hat{\mathbf{F}}$ as follows:

$$
\begin{aligned}
\overline{\mathbf{F}} & =\left[f_{1 \ldots}^{i} f_{1}^{n} f_{2 \ldots}^{i} f_{2}^{n} f_{3 \ldots}^{i} f_{3}^{n} f_{4 \ldots}^{i} f_{4}^{n} f_{6 \ldots}^{i} f_{6}^{n} f_{8 \ldots}^{i} f_{8}^{n}\right]^{T} \\
\hat{\mathbf{F}} & =\left[f_{5 \ldots}^{i} f_{5}^{n} f_{7 \ldots}^{i} f_{7}^{n}\right]^{T}
\end{aligned}
$$

Now, substitute equations (13) and (24) into the variational equation (7), and perform integration over the thickness direction $\zeta$, as $g_{l}^{i}(\zeta)$ are known. The coefficients of $\delta f_{l}^{i}$ in the resulting expression are individually equated to zero, since the variations are arbitrary. This yields the following $8 n$ differentialalgebraic equations for unknowns $f_{l}^{i}$ :

$$
\begin{aligned}
& \mathbf{N} \overline{\mathbf{F}}_{, \xi}=\overline{\mathbf{B}} \overline{\mathbf{F}}+\hat{\mathbf{B}} \hat{\mathbf{F}}+\overline{\mathbf{P}} \\
& \mathbf{L} \hat{\mathbf{F}}=\hat{\mathbf{B}}(\xi) \overline{\mathbf{F}}+\tilde{\mathbf{P}}
\end{aligned}
$$

where $\mathbf{N}, \overline{\mathbf{B}}, \hat{\mathbf{B}}, \mathbf{L}$ and $\tilde{\mathbf{B}}$ are matrices of size $6 n \mathrm{x}$ $6 n, 6 n \times 6 n, 6 n \times 2 n, 2 n \times 2 n$ and $2 n \times 6 n$, respectively. The nonzero elements of these matrices are as given in Dhanesh et al. (2016), and are omitted here for brevity. $\overline{\mathbf{P}}$ and $\tilde{\mathbf{P}}$ represent the load vectors of size $6 n$ and $2 n$, and their nonzero elements obtained as

$$
\begin{aligned}
\bar{P}_{i 1}= & \varepsilon_{0}\left\langle\tilde{s}_{21} g_{4}^{i}\right\rangle_{h}-\chi_{0}\left\langle\tilde{s}_{21}\left(z_{k-1}+t \zeta\right) g_{4}^{i}\right\rangle_{h} \\
\bar{P}_{i 2}= & \varepsilon_{0}\left\langle\tilde{s}_{26} g_{6}^{i}\right\rangle_{h}-\chi_{0}\left\langle\tilde{s}_{26}\left(z_{k-1}+t \zeta\right) g_{6}^{i}\right\rangle_{h} \\
& +\Theta\left\langle\left(z_{k-1}+t \zeta\right) g_{6}^{i}\right\rangle_{h} \\
\tilde{P}_{i 1}= & -\varepsilon_{0}\left\langle\tilde{s}_{23} g_{5}^{i}\right\rangle_{h}+\chi_{0}\left\langle\tilde{s}_{23}\left(z_{k-1}+t \zeta\right) g_{5}^{i}\right\rangle_{h}
\end{aligned}
$$




$$
\tilde{P}_{i 2}=\Theta a \xi\left\langle g_{7}^{i}\right\rangle_{h}
$$

where the notation $\langle\ldots\rangle_{h}=\sum_{k=1}^{L} t^{(k)} \int_{0}^{1}(\ldots)^{(k)} d \zeta$ represents the integration across the thickness of the laminate. Similar to the first step, all integrals in nonzero terms of the above matrices are evaluated exactly in closed form. Since the resulting differentialalgebraic system of equations in this step ((26) and (27)) are of same nature as in the previous step ((17) and (18)), the solution procedure remains the same. The unknown constants are obtained from the free edge boundary conditions obtained from Eq. (10), written in terms of $f_{l}^{i}$ as

$$
f_{8}^{i}=0, f_{4}^{i}=0, f_{6}^{i}=0, i=1,2, \ldots, n
$$

The solution of $\overline{\mathbf{F}}(\xi)$ and $\hat{\mathbf{F}}(\xi)$ completes the second step, and one iteration in the solution process.

The steps for computing $g_{l}^{i}(\zeta)$ and $f_{l}^{i}(\xi)$ are repeated until desired level of convergence is achieved. For a particular problem, the convergence study is performed by obtaining the solution using different number of terms $(n)$ in the solution approximation. From the previous studies on composite laminates, it has been observed that the solution for each term converges within two to three iterations, and in most of the problems, an accurate solution can be obtained with five to six terms. The convergence of the MMEKM solution for free edge problems in sandwich laminate will be verified in the numerical results section.

\section{Numerical Results and Discussions}

Numerical results are presented for the free edge stress field in soft-core sandwich panels with the layup configurations as shown in Fig. 2. Five-layer sandwich panels having a thick central core and two

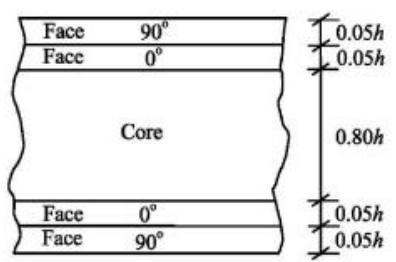

(a) Cross-ply sandwich panel

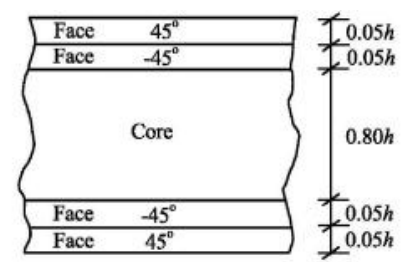

(b) Angle-ply sandwich panel
Fig. 2: Lay-ups of sandwich panel

thin composite face sheets at its bottom and top with cross-ply [90/0/core $]_{s}$ and angle-ply [45/-45/core $]_{s}$ layups are considered. The material properties of the soft-core and face sheet layers are selected from Kapuria and Achary (2006) and are presented in Table 1. The span-to-thickness ratio of the panel considered is $S=a / h=5$. The numerical results are presented for the extension $\left(\varepsilon_{0}\right)$, bending $\left(\chi_{0}\right)$ and twisting $(\Theta)$ load cases. The results are normalized with respect to the corresponding load as follows:

Extension:

$$
\left(\bar{\sigma}_{z}, \bar{\tau}_{y z}, \bar{\tau}_{z x}\right)=\left(\sigma_{z}, \tau_{y z}, \tau_{z x}\right) S / Y_{2} \varepsilon_{0}
$$

Bending:

$$
\left(\bar{\sigma}_{z}, \bar{\tau}_{y z}, \bar{\tau}_{z x}\right)=\left(\sigma_{z}, \tau_{y z}, \tau_{z x}\right) S^{2} / a Y_{2} \chi_{0}
$$

Twisting:

$$
\left(\bar{\sigma}_{z}, \bar{\tau}_{y z}, \bar{\tau}_{z x}, \bar{\tau}_{x y}\right)=\left(\sigma_{z}, \tau_{y z}, \tau_{z x}, \tau_{x y}\right) S / a Y_{2} \Theta
$$

A dimensionless global thicknesses coordinate $\bar{\zeta}=z / h$ varying from -0.5 to 0.5 is introduced to present the through-thickness distributions of stresses.

\section{Uniform Extension}

First, the cross-ply sandwich panel shown in Fig. 2(a) under unit axial strain is considered. Fig. 3 shows the longitudinal distributions of the interlaminar transverse

Table 1: Material Properties

\begin{tabular}{lccccccccc}
\hline Material & $\mathrm{Y}_{1}$ & $\mathrm{Y}_{2}$ & $\mathrm{Y}_{3}$ & $\mathrm{G}_{12}$ & $\mathrm{G}_{23}$ & $\mathrm{G}_{31}$ & $v_{12}$ & $v_{13}$ & $v_{23}$ \\
\hline & & & $(\mathrm{GPa})$ \\
Face $^{1}$ & 131.0 & 6.9 & 6.9 & 3.588 & 2.3322 & 3.588 & 0.32 & 0.32 & 0.49 \\
Core $^{1}$ & 0.2208 & 0.2001 & 2.76 & 0.01656 & 0.4554 & 0.5451 & 0.99 & $3 \times 10^{-5}$ & $3 \times 10^{-5}$ \\
\hline
\end{tabular}

${ }^{1}$ Kapuria and Achary (2006) 

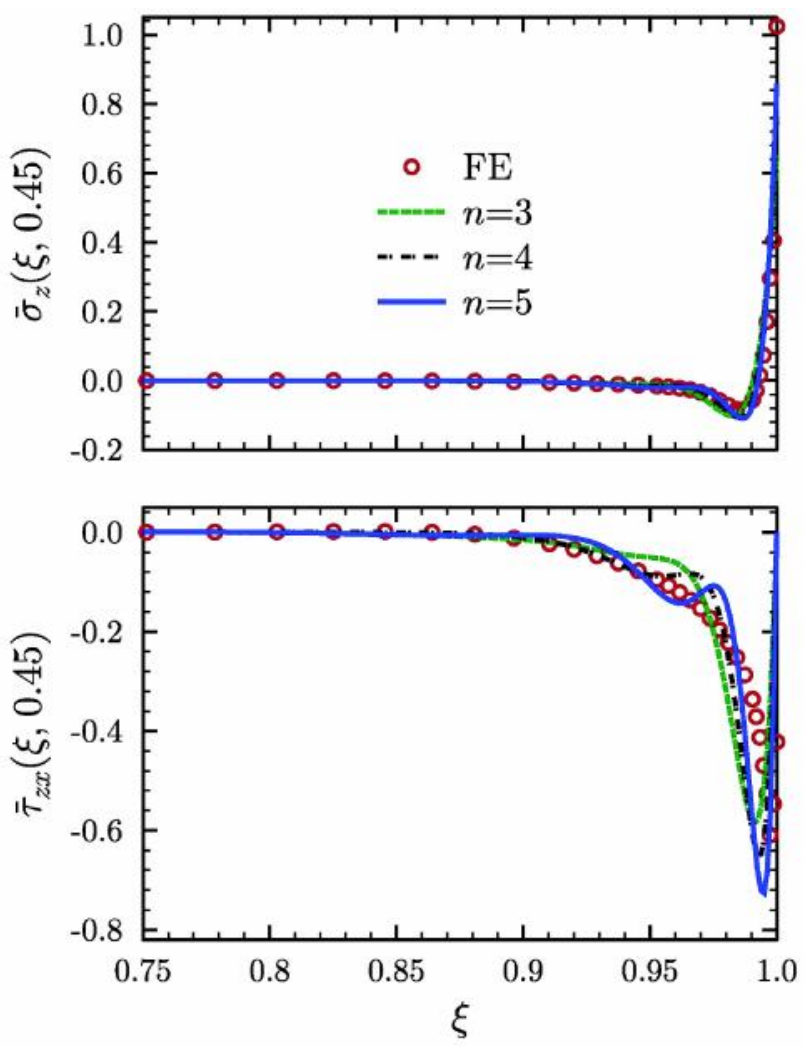

Fig. 3: Distributions of $\sigma_{z}$ and $\tau_{z x}$ along the $0 / 90$ interface of cross-ply sandwich $[90 / 0 / \text { core }]_{\mathrm{s}}$ panel under uniform extension

stresses, $\bar{\sigma}_{z}$ and $\bar{\tau}_{z x}$, at the $0 / 90$ interface obtained using different number of terms $n$. The convergence for a given number of terms has been found to occur within two to three iterations. Accordingly, the results corresponding to three iterations are shown here. The solution obtained with different number of terms also shows good convergence along the width of the laminate. It can be observed that $\bar{\sigma}_{z}$ at the free edge and peak value of $\bar{\tau}_{z x}$ continue to rise as $n$ increases, indicating a singular nature of these variables. To verify the distribution of converged MMEKM solution, the present results are compared with the detailed finite element solution obtained using the commercial software, ABAQUS 6.12, as there are no published results available in the literature for 3D elasticity based free edge stress field in sandwich laminates under extension loading. The layers are modeled using the four-node linear generalized plane strain quadrilateral element (CPEG4R) with reduced integration. In order to capture the sharp variation of stresses near the free edge, the half width of the panel is modelled with 100 elements and the mesh is refined towards the edges with a bias ratio of 10 . In the thickness direction, the face sheet and core layer are modeled with 10 and 40 elements, respectively. Fig. 3 shows that the distributions of $\bar{\sigma}_{z}$ and $\bar{\tau}_{z x}$ obtained using the MMEKM are in excellent agreement with the FE solution. In the present solution, the free edge traction free condition can be seen to be satisfied exactly. In the FE solution, however, large nonzero stresses are observed at the free edge, which could affect the accuracy of free edge stress prediction. The throughthickness distributions of peel stress $\bar{\sigma}_{z}$ at the free edge obtained with different values of $n$ are shown along with the FE solution in Fig. 4. The MMEKM solution shows good convergence and is generally in agreement with the FE solution. Note that FE solution does not satisfy the continuity of transverse stresses at the layer interfaces. The interlaminar free edge peel stress is concentrated near the interface between the face sheet layers and is singular in nature.

The angle-ply sandwich [45/-45/core $]_{s}$ panel shown in Fig. 2(b) is analyzed next under a unit axial strain $\varepsilon_{0}=1$. The longitudinal variations of $\bar{\tau}_{y z}$ and $\bar{\sigma}_{z}$ (Fig. 5) obtained with different values of $n$ show excellent convergence, with the solutions for $n=5$ and $n=6$ almost coinciding with each other. The interlaminar stresses show sharp variation near the free edge at the interface between the two face sheet layers. The through-thickness distribution of $\bar{\tau}_{y z}$ at the free edge presented in Fig. 6 further verifies the convergence of the present solution. $\bar{\tau}_{y z}$ is almost

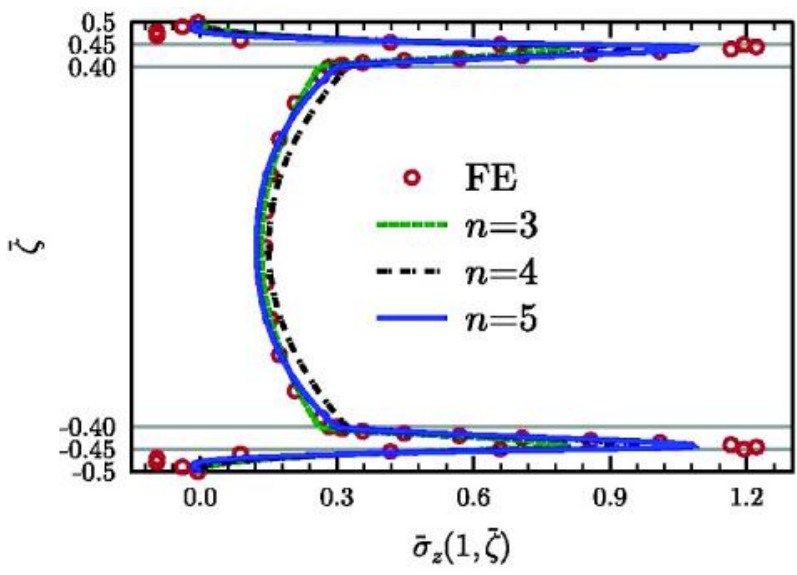

Fig. 4: Through-thickness distribution of $\sigma_{z}$ at the free edge of cross-ply sandwich $\left[90 / 0 /\right.$ core $_{s}$ panel under uniform extension 

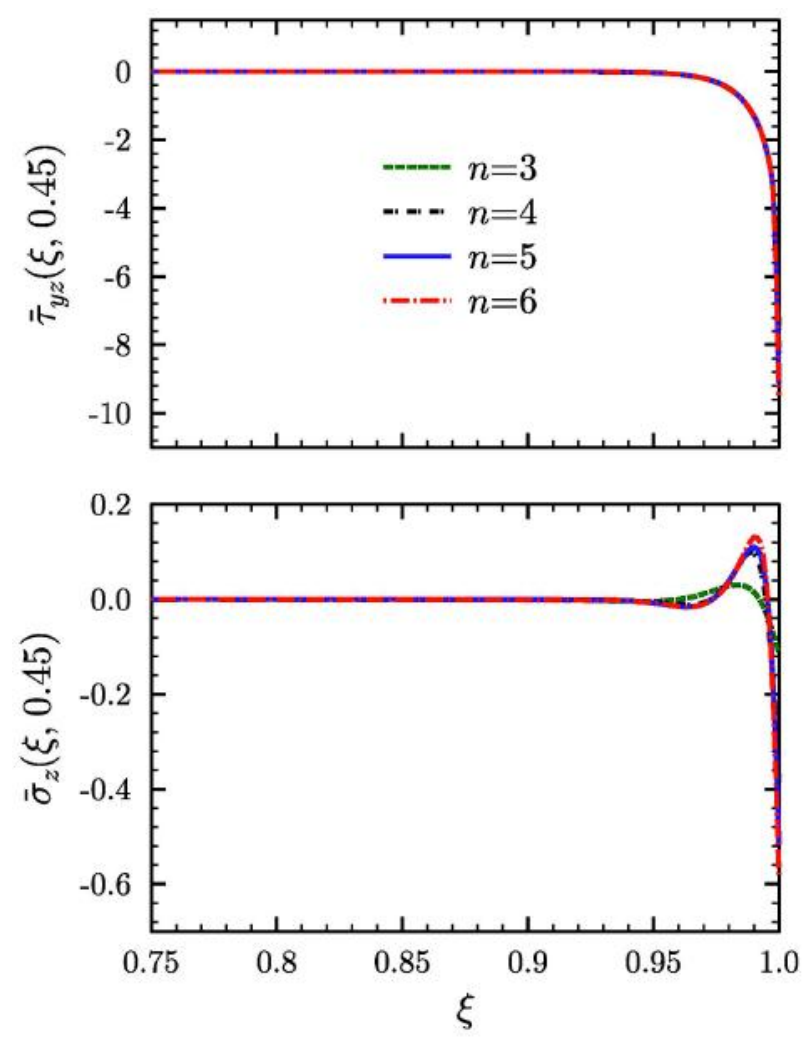

Fig. 5: Distributions of $\tau_{y z}$ and $\sigma_{z}$ along the $-45 / 45$ interface of angle-ply sandwich $[45 /-45 / \text { core }]_{s}$ panel under uniform extension

absent in the interior part of the soft core, and is concentrated at the interface between the face sheet layers, making this interface more prone to delamination than the face sheet-soft core interface. A close observation of $\bar{\tau}_{y z}$ at the $-45 / 45$ interface

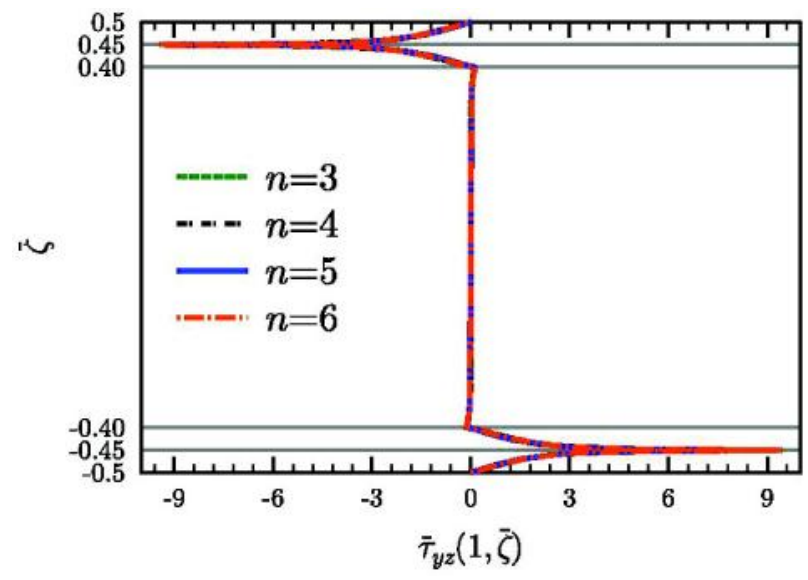

Fig. 6: Through-thickness distribution of $\tau_{y z}$ at the free edge of angle-ply sandwich $[45 /-45 / \text { core }]_{s}$ panel under uniform extension $(\bar{\zeta}=0.45)$ reveals that it is singular in nature with its peak value growing with $n$.

\section{Bending}

The free edge effects in sandwich panels under the unit bending curvature $\left(\chi_{0}=1\right)$ loading is studied in this section. Fig. 7 shows the distributions of the interlaminar transverse stresses, $\bar{\sigma}_{z}$ and $\bar{\tau}_{z x}$ at the $0 /$ 90 interface of the sandwich panel for different number of terms $n$. Similar to the extension loading, the interlaminar stresses show excellent convergence for $n \geq 4$. As before, $\bar{\sigma}_{z}$ at the free edge shows a gradual increase in its peak with the use of higher terms, indicating its singular character. The peak value of $\bar{\tau}_{z x}$ also shows a similar trend. In absence of any solution available in literature, the present solution is compared with the detailed FE solution of ABAQUS, and they show good agreement (Fig. 7). The throughthickness distribution of $\bar{\sigma}_{z}$ at the free edge presented in Fig. 8, shows high concentration of the peel stress
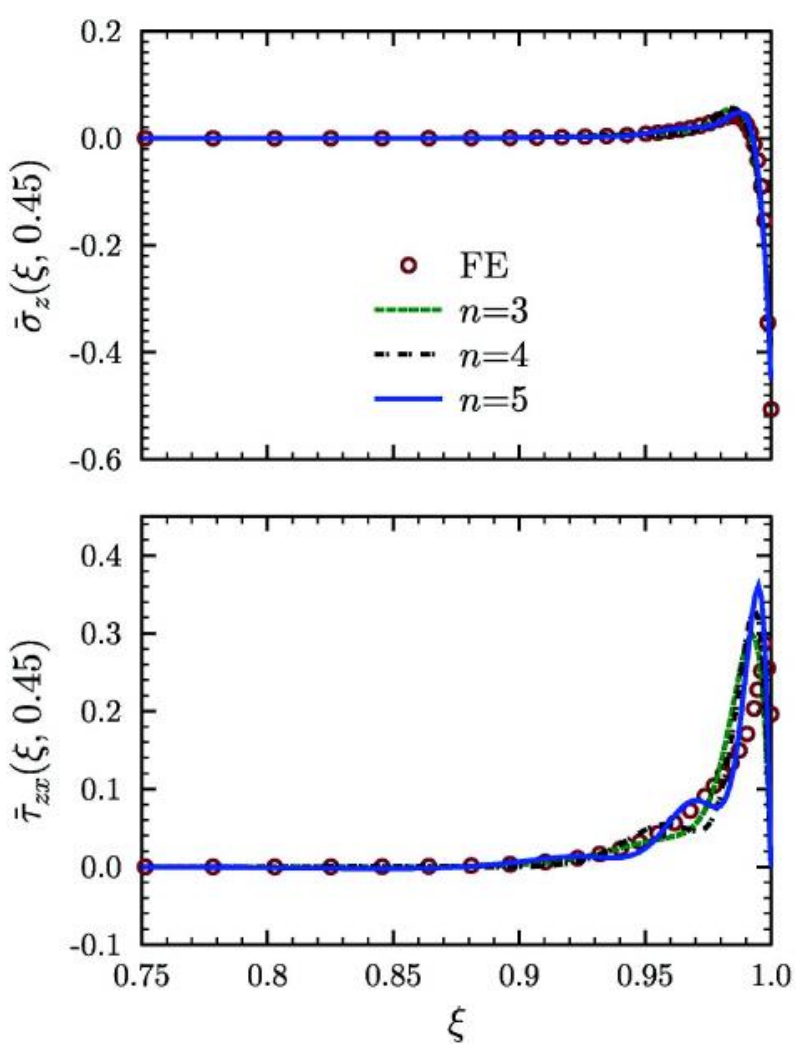

Fig. 7. Distributions of interlaminar stresses $\sigma_{z}$ and $\sigma_{z x}$ along the $0 / 90$ interface of cross-ply sandwich $[90 / 0 / \text { core }]_{s}$ panel under bending 
near the interface between face sheet layers, similar to the extension load case.

Having established the validity of the MMEKM solution for cross-ply sandwich in comparison with the FE solution, further results are presented for angle-

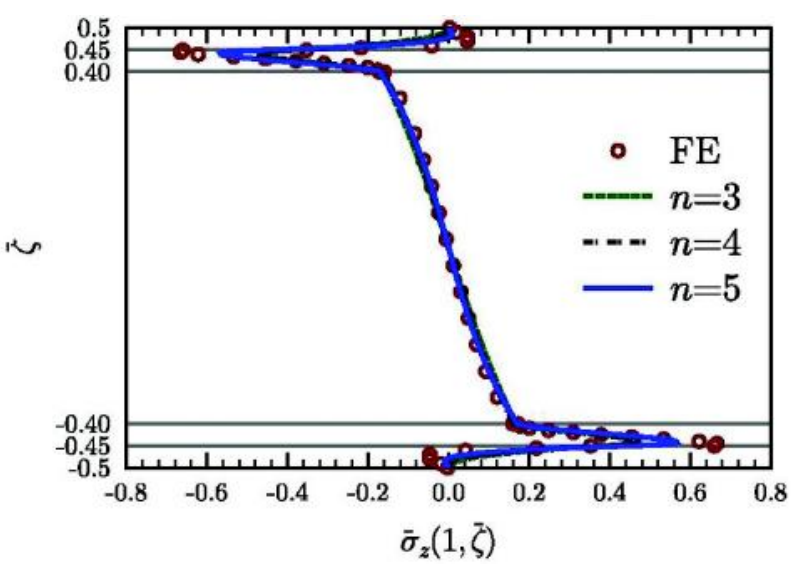

Fig. 8: Through-thickness distribution of $\sigma_{z}$ at the free edge for cross-ply sandwich $[90 / 0 / \text { core }]_{s}$ panel under bending
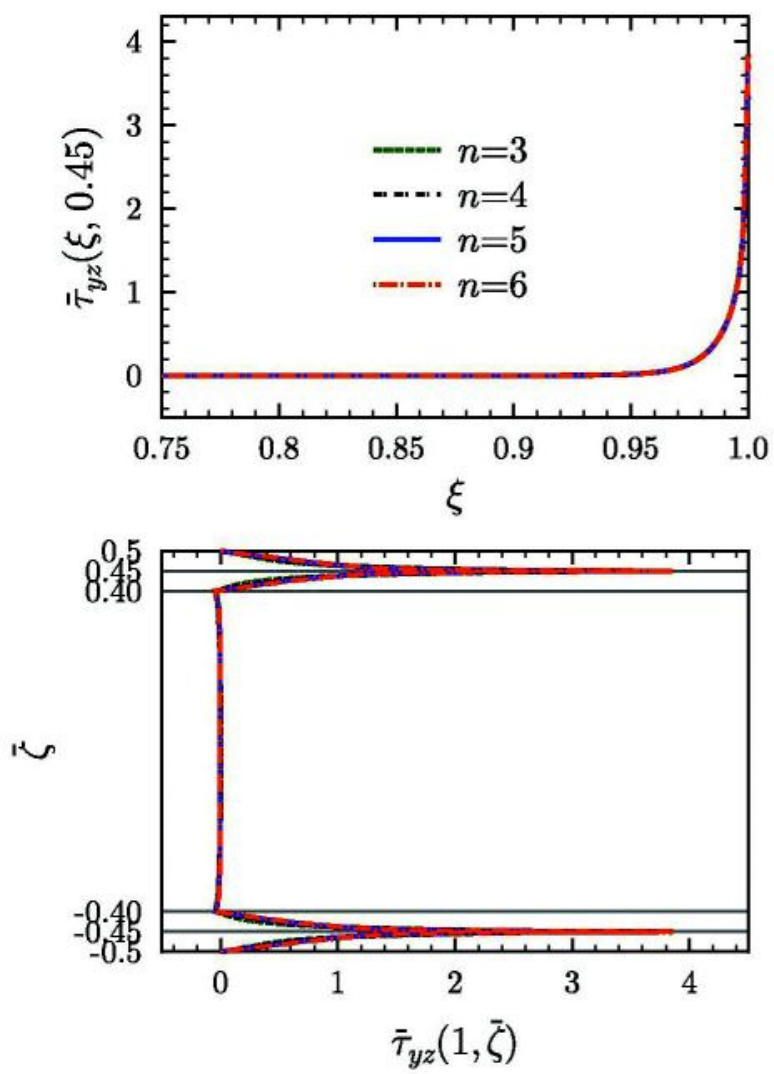

A ply sandwich $[45 /-45 / \text { core }]_{s}$ panel under the bending curvature loading. Fig. 9(A) shows the longitudinal distribution of $\bar{\tau}_{y z}$ at the $-45 / 45$ interface along with its through thickness-distribution at the free edge. Figure 9 (B) shows the distributions of $\bar{\sigma}_{z}$ and $\bar{\tau}_{z x}$ at the $-45 / 45$ interface of the sandwich panel. Once again, the MMEKM solution converges with $n \geq 4$, and successfully captures the singular nature of $\bar{\sigma}_{z}$ and $\bar{\tau}_{z x}$ near the free edge region. The throughthickness distribution of $\bar{\tau}_{y z}$ at the free edge shows a large stress concentration at the interface between face sheet layers for this loading too.

\section{Twisting}

In this section, the sandwich panels are analyzed for unit twisting curvature $(\Theta=1)$ loading. The longitudinal and through-thickness distributions of $\bar{\tau}_{y z}$ for the crossply sandwich panel presented in Fig. 10 show a faster
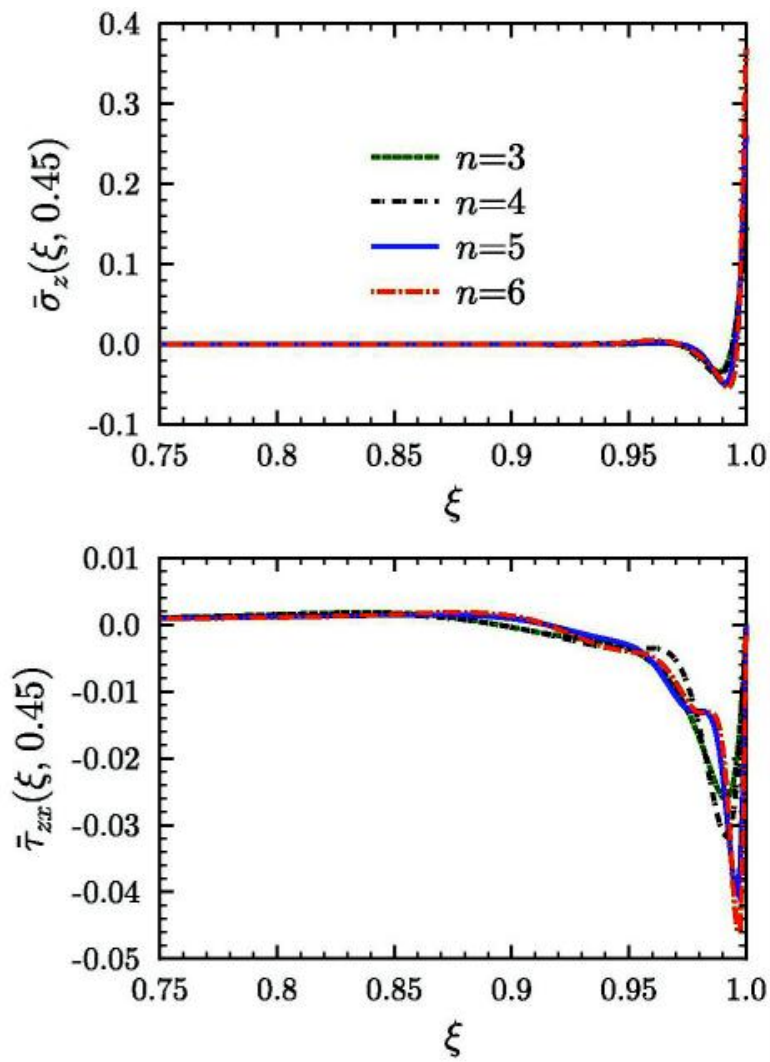

B

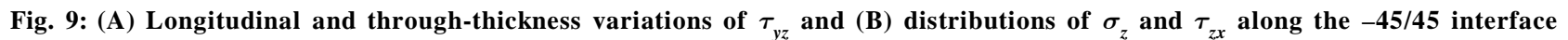
for angle-ply sandwich panel $[45 /-45 / \text { core }]_{s}$ under bending 

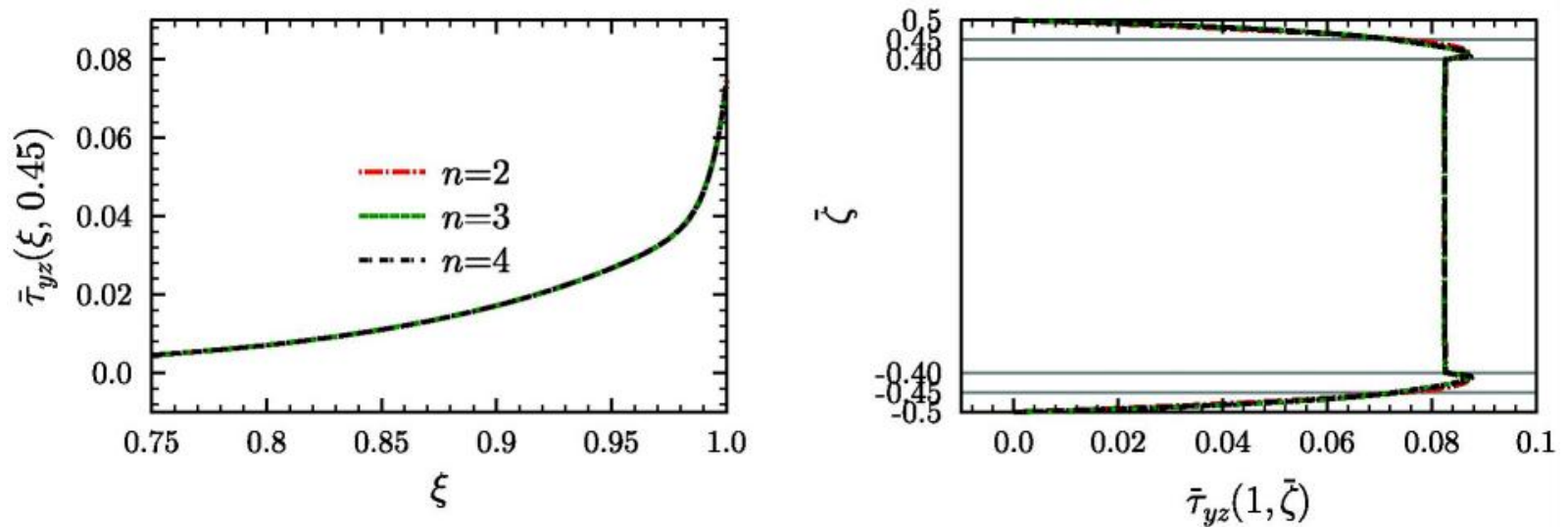

Fig. 10: Longitudinal (along 0/90 interface) and through-thickness (at the free edge) distributions of $\tau_{z x}$ for cross-ply sandwich $[90 / 0 / \text { core }]_{s}$ panel under twisting
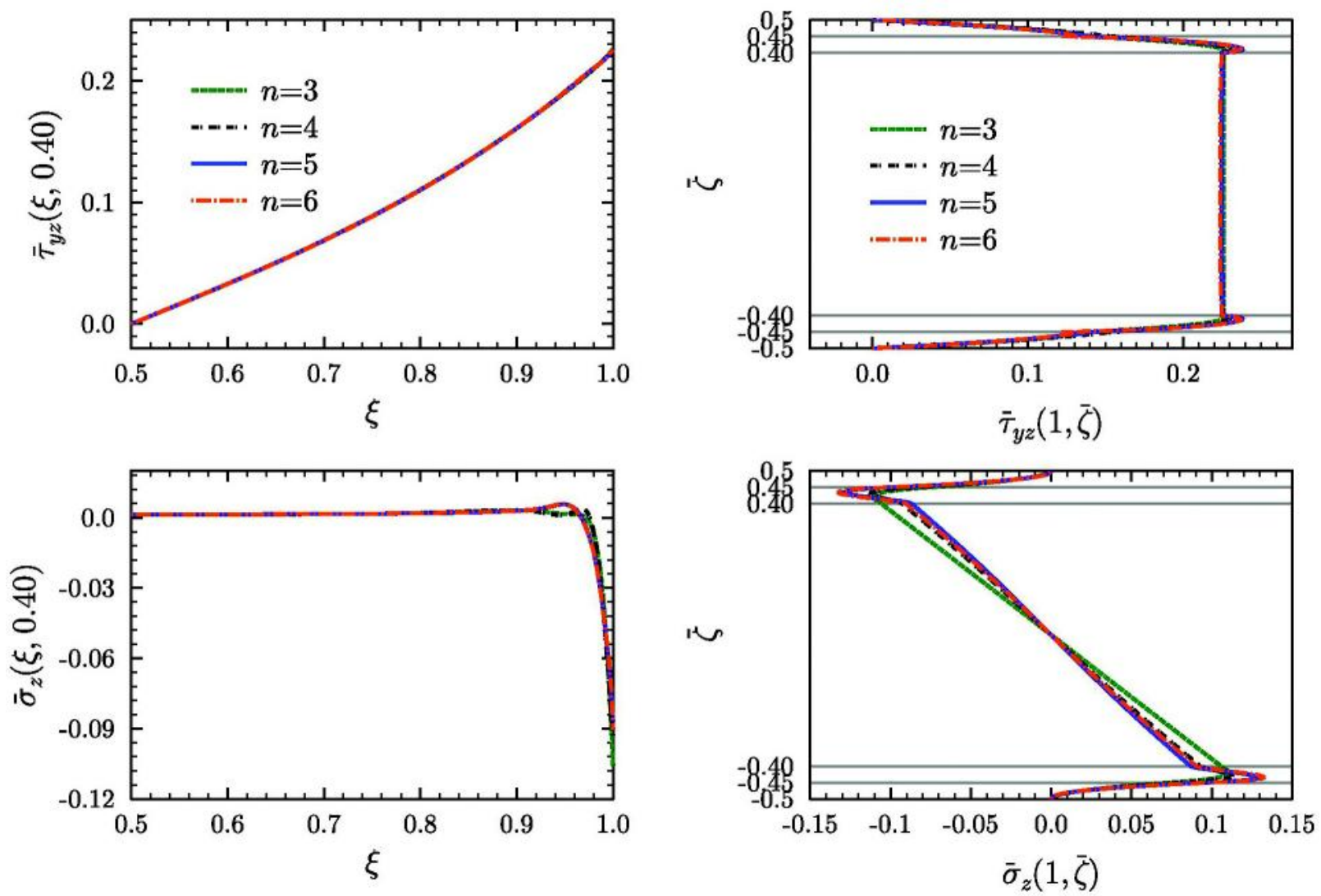

A

B

Fig. 11: (A) Longitudinal variations of $\tau_{y z}$ and $\sigma_{z}$ along the $-45 / 45$ interface and (B) through-thickness distributions of $\tau_{y z}$ and $\sigma_{z}$ at the free edge for angle-ply sandwich $[45 /-45 / \text { core }]_{s}$ under twistingConclusions

convergence with $n \geq 3$ for twisting loading, and no singularity is observed at the free edge interface. The variations of interlaminar stresses $\bar{\tau}_{y z}$ and $\bar{\sigma}_{z}$ along the interface between face sheet and soft core shown in Fig. 11(A) for the angle-ply sandwich panel also confirm the rapid convergence of the solution under the twisting loading. The through-thickness variations of $\bar{\tau}_{y z}$ at the free edge of cross-ply (Fig. 10) and angle-ply (Fig. 11(B)) sandwich panel reveal that, under twisting loading, its maximum value occurs near 
the face sheet-soft core interface and is not singular. Also the longitudinal variation of $\bar{\tau}_{y z}$ is not as sharp as observed in extension and bending cases. Fig. 11(B) also shows that the maximum value of $\bar{\sigma}_{z}$ in the angleply sandwich laminate occur in the interior of the $45^{\circ}$-ply near the $-45 / 45$ interface.

\section{Conclusions}

An accurate analytical solution for the free edge stress field in sandwich laminates under extension, bending and twisting loadings is presented. The governing equations for the free edge problem is developed using the Reissner-type variational principle. The analytical solution for the governing equations is obtained iteratively using the MMEKM, recently developed by the author group. The mixed-field approach considers both displacements and stresses as unknown variables, and ensures the exact point-wise satisfaction of free edge and interlaminar conditions. Numerical results

the core-face sheet interface for the twisting loading.

\section{References}

Afshin M Sadighi M. and Shakeri M (2010) Free-edge effects in a cylindrical sandwich panel with a flexible core and laminated composite face sheets Mech Compos Mater 46 539-554

Andakhshideh A and Tahani M (2013a) Free-edge stress analysis of general rectangular composite laminates under bending, torsion and thermal loads Eur J Mech A-Solids 42 229-240

Andakhshideh A and Tahani M (2013b) Interlaminar stresses in general thick rectangular laminated plates under in-plane loads Compos Part B-Eng 47 58-69

Cho M and Kim H S (2000) Iterative free-edge stress analysis of composite laminates under extension, bending, twisting and thermal loadings Int J Solids Struct 37 435-459

Cho M and Yoon J Y (1999) Free-edge interlaminar stress analysis of composite laminates by extended Kantorovich method AIAA J 37 656-660

Davi G and Milazzo A (1999) Bending stress fields in composite laminate beams by a boundary integral formulation Comput Struct 71 267-276

Dhanesh N Kapuria S and Achary G G S (2015) Accurate prediction of three-dimensional free edge stress field in composite laminates using mixed-field multiterm extended are presented for sandwich laminates having face sheets with cross-ply and angle-ply lay-ups, subjected to uniform extension, bending and twisting loading. The presented numerical results reveal that, similar to composite laminates, the MMEKM solution for the free edge stress field in sandwich laminates also shows rapid convergence within four to five terms, and within three iterations for all loadings and lay-ups studied here. The converged MMEKM solution shows good agreement with the detailed FE solution. The present solution successfully captures the singularity of stresses at its peak location both in crossply and angle-ply sandwich panels under extension and bending loadings. In case of twisting loading, the MMEKM solution converges faster within three terms. The free edge stresses obtained for both the lay-ups under the twisting loading converge to a finite value, showing its nonsingular nature. The transverse shear stresses at the free edge are found to be maximum near the interface between face sheet layers for extension and bending loadings, and near

\section{Kantorovich method Acta Mech 1-25}

Flanagan G (1994) An efficient stress function approximation for free-edge stresses in laminates Int J Solids Struct 31 941952

Hayashi T (1967) Analytical study of interlaminar shear stresses in a laminated composite plate Trans Jpn Soc Aeronaut Space Sci 10 43-48

Jones R M (1999) Mechanics of composite materials Taylor \& Francis New York

Kant T and Swaminathan K (2000) Estimation of transverse/ interlaminar stresses in laminated composites - a selective review and survey of current developments Compos Struct 49 65-75

Kapuria S and Achary G G S (2006) Nonlinear zigzag theory for electrothermo-mechanical buckling of piezoelectric composite and sandwich plates Acta Mech 184 61-76

Kapuria S and Kumari P (2011) Extended Kantorovich method for three-dimensional elasticity solution of laminated composite structures in cylindrical bending J Appl Mech ASME 78 Art no. 061004

Kerr A D (1968) An extension of the Kantorovich method QAppl Math 26 219-229

Lekhnitskii S G (1963) Theory of elasticity of an anisotropic elastic body holden - Day San Francisco 
Lessard L B Schmidt A S and Shokrieh M M (1996) Threedimensional stress analysis of free-edge effects in a simple composite cross-ply laminate Int J Solids Struct 33 22432259

Lindemann $\mathbf{J}$ and Becker W (2000) Analysis of the free-edge effect in composite laminates by the boundary finite element method Mech Compos Mater 36 207-214

Lovinger Z and Frostig Y (2004) High order behaviour of sandwich plates with free edges-edge effects Int J Solids Struct $\mathbf{4 1}$ 979-1004

Mittelstedt C and Becker W (2004) Interlaminar stress concentrations in layered structures: Part I-A selective literature survey on the free-edge effect since 1967 J Compos Mater 38 1037-1062

Mittelstedt C and Becker W (2007) Free-edge effects in composite laminates Appl Mech Rev 60 217-244

Pipes R B and Pagano N J (1970) Interlaminar stresses in composite laminates under uniform axial extension $J$
Compos Mater 4 538-548

Puppo A H and Evensen H A (1970) Interlaminar shear in laminated composites under generalized plane stress $J$ Compos Mater 4 204-220

Raju I S and Crews J H (1981) Interlaminar stress singularities at a straight free edge in composite laminates Comput Struct 14 21-28

Shames I H and Dym C L (1985) Energy and finite element methods in structural mechanics Hemisphere Publishing New York

Wang A S D and Crossman F W (1977) Some new results on edge effect in symmetric composite laminates J Compos Mater 11 92-106

Wong E H (2015) Interfacial stresses in sandwich structures subjected to temperature and mechanical loads Compos Struct 134 226-236

Wong E H (2016) Design analysis of sandwiched structures experiencing differential thermal expansion and differential free-edge stretching I J Adhes Adhes 65 19-27. 